\title{
Quantum Logics and Convex Geometry
}

\author{
L. J. Bunce and J. D. Maitland Wright \\ Department of Mathematics, University of Reading, Whiteknights, Reading RG6 2AX, England
}

\begin{abstract}
The main result is a representation theorem which shows that, for a large class of quantum logics, a quantum logic, $Q$, is isomorphic to the lattice of projective faces in a suitable convex set $K$. As an application we extend our earlier results [4], which, subject to countability conditions, gave a geometric characterization of those quantum logics which are isomorphic to the projection lattice of a von Neumann algebra or a $J B W$-algebra.
\end{abstract}

\section{Introduction}

Quantum logics, alias complete orthomodular lattices, arise in mathematical models of quantum mechanics as the lattice of "questions." The completely additive probability measures on a quantum logic correspond to the physical states of a quantum mechanical system. When $M$ is a von Neumann algebra or a $J B W$-algebra then $P(M)$, the lattice of projections in $M$, is a quantum logic. For mathematical convenience, rather than any compelling physical reason, it is frequently assumed that the quantum logic of a mechanical system is $P(M)$. So it may be of some physical as well as mathematical interest to determine when a given quantum logic is isomorphic to a lattice of projections, $P(M)$.

Atomic lattices have been very thoroughly investigated by a number of authors and, in particular, key results were obtained by Piron and Wilbur. For a detailed discussion and a full list of references, the reader is referred to [11]. Using projective geometry, Wilbur characterized $P(L(H))$ by lattice conditions, [11]. Unfortunately, these methods are not applicable to non-atomic lattices. One way of coping with this difficulty is to focus attention on the geometry of the convex set of completely additive probability measures (physical states). Using this approach, we characterised the lattices $P(M)$ where $M$ is a von Neumann algebra or a $J B W$-algebra, up to isomorphism, subject to certain countability conditions, see [4]. We made use of a deep theorem of Iochum and Shultz, [9], on the geometry of the normal state spaces of von Neumann algebras and $J B W$-algebras, together with the GleasonChristensen-Yeadon theorem $[5,12]$ and its extension to $J B W$-algebras [3].

Our results here show that a very large class of quantum logics may be identified with lattices of "projections" arising as natural geometric objects in certain convex 
sets. It is hoped that the general structural results we obtain will be useful for future investigations. One application of these results, given in Corollary 9, is a geometric characterisation of those logics which are isomorphic to the lattice of all projections in a von Neumann algebra of a $J B W$-algebra.

\section{Preliminaries}

Let $L$ be a complete orthomodular lattice with orthocomplementation, $\perp$. As usual, we denote the least and largest elements of $L$ by 0,1 , respectively. The elements $x, y$ of $L$ are said to be disjoint (said by some authors to be orthogonal) if $x \leqq y^{\perp}$. A completely additive measure on $L$ is a non-negative real-valued function, $\phi$ : $L \rightarrow R$, with $\phi(0)=0$, such that $\phi\left(\vee x_{\alpha}\right)=\Sigma \phi\left(x_{\alpha}\right)$, for every family of mutually disjoint elements, $\left(x_{\alpha}\right)$, in $L$. If, in addition, $\phi(1)=1$, then $\phi$ is said to be a probability measure. The set of all completely additive probability measures on $L$ is a $\sigma$-convex set, denoted by $K_{L}$.

Consider a convex subset $K$ of $K_{L}$, satisfying the conditions

(1): Given $x, y$ in $L$, then $x \leqq y$ if and only if $\{\phi \in K ; \phi(x)=1\} \subseteq\{\phi \in K$; $\phi(y)=1\}$.

(2): If $x, y \in L, \phi \in K$ and $\phi(x)=\phi(y)=1$, then $\phi(x \wedge y)=1$.

Letting $V(K)$ be the (real) linear space generated by $K$, it is easy to see that $V(K)=$ cone $K$ - cone $K$ and that the Minkowski functional over $\operatorname{co}(K \cup-K)$ is a norm. Thus $V(K)$ is a base-norm space with base $K$ (though, in general, the closed unit ball will not equal $c o(K \cup-K))$. The dual normed linear space $V(K)^{*}$ is an order unit space in the natural ordering, with closed unit ball $[-1(K), 1(K)]$, where $1(K)$ is the order unit (thus the norm on $V(K)^{*}$ coincides with the induced order unit norm). Thus one obtains an order preserving linear isometry $V(K)^{*} \rightarrow$ $A^{b}(K)\left(\left.f_{\mapsto} f\right|_{K}\right)$. Further, any given $b_{0} \in A^{b}(K)$ extends uniquely to a linear functional, $b$, on $V(K)$, defined by $b(\phi)=\lambda b_{0}(\sigma)-\mu b_{0}(\tau)$, whenever $\phi=\lambda \sigma-v \tau$, where $\lambda, \mu \geqq 0, \sigma, \tau \in K$. Since $\|\phi\|=\inf \{\lambda+\mu, \phi=\lambda \sigma-\mu \tau$, where $\lambda, \mu \geqq 0$, $\sigma, \tau \in K\}$, it follows that $b \in V(K)^{*}$, and of course $b_{0}=\left.b\right|_{K}$. Therefore, $V(K)^{*} \simeq A^{b}(K)$.

With each $x$ in $L$, there is associated an element $\hat{x}$ in $[0,1(K)] \subseteq V(K)^{*}$, determined by $\hat{x}(\phi)=\phi(x)$, for every $\phi$ in $K$. The set $\hat{L}=\{\hat{x} ; x \in L\}$ has a natural structure as a complete orthomodular lattice, induced by $L$, and satisfies (1) and (2), and also $L \simeq \hat{L}$. Henceforth, we will identify $L$ with $\hat{L}$. Note that the orthocomplementation then becomes, $x^{\perp}=1-x$, where $x \in L$ and $1=1(K)$. It will aid clarity if, throughout, we keep to the notation $\phi(a)$ (instead of $\hat{a}(\phi)$ or $\langle a, \phi\rangle)$ whenever $d \in V(K)^{*}, \phi \in V(K)$.

In order to be consistent with [4], we shall say that $K$ is strongly full if, together with (1) and (2) the following condition

(3): Whenever $\phi \subset K$ is faithful on $L$ then $\phi$ is also faithful on $V(K)^{*+}$, is also satisfied.

There is another condition, the Jordan-Hahn property, which is closely connected with strong-fullness. It is said that $K$ has the Jordan-Hahn property if for each $\rho$ in $V(K)$ there exist $\sigma, \tau$ in $K, x$ in $L$, and real numbers $\lambda, \mu \geqq 0$, 
for which

$$
\rho=\lambda \sigma-\mu \tau, \quad \sigma(x)=\tau(1-x)=1 .
$$

It is a point of some interest to decide when $L$ is a subset of the extreme points, ext $[0,1]$, of the unit interval of $V(K)^{*}$ and, in particular when $L$ is equal to interesting subsets of ext $[0,1]$, such as the set of weak* exposed points, exp $[0,1]$. (In connection with these remarks, and also for the Jordan-Hahn property, see [10], where the particular case of finite orthogonality spaces is considered.) We are able to answer these questions in a very general setting in Lemma 2 . In passing let us recall that the point, $p$, in a convex subset, $C$, of $V(K)^{*}$ is a (weak*) exposed point of $C$ if there exists $\rho \in V(K)$ which takes its greatest value in $C$ only at the point $p$.

We now turn to the particular convex sets in which we are interested. Alfsen and Shultz in [1], and particularly in [2], investigate those (radially compact) convex sets, $K$, which are the distinguished base of a base norm space $V$, with $V^{*}=A^{b}(K)$, and for which every norm-exposed face of $K$ is projective. Let us agree to call such convex sets projective convex sets. (This should not lead to confusion, for by [2, Proposition 1.10], every projective face of $K$ will be projective as a convex set, according to this terminology). The reader is referred to [1] and [2] for the meaning of unexplained terms used here, and after.

The projective units, $\mathbb{P}$, of $A^{b}(K)$, where $K$ is projective lie in (but, possibly are not equal to) ext $[0,1]$, where 1 is the order unit of $A^{b}(K)$, and they form a complete orthomodular lattice isomorphic to the lattice of $P$-projections on $A^{b}(K)$, and the lattice of projective faces of $K$. For each $P$-projection, $P$, on $A^{b}(K), P(1) \in \mathbb{P}$, and every projective unit is (uniquely) of this form. The correspondence between $\mathbb{P}$ and the projective faces of $K$ is given by, $p \rightarrow F_{p}=\{\phi \in K ; \phi(p)=1\}$. Moreover, if $\left(p_{\lambda}\right)$ is any family in $\mathbb{P}$, then $\wedge F_{p_{\lambda}}=\cap F_{p_{\lambda}}=F_{\wedge p_{\lambda}}$ (by [2, Corollary 1.2]). In addition, with each $a$ in $A^{b}(K), 0 \leqq a \leqq 1$, there exists a least element, $r(a)$, in $P$, with $a \leqq r(a)$; and, given $\phi \in K, \phi(a)=0$ if and only if $\phi(r(a))=0$. Note that $A^{b}(K)$ is monotone complete, and that a simple limit argument shows that $K \subseteq K_{\mathrm{p}}$.

It is important to notice that $K$ satisfies conditions (1), (2) and (3) (i.e. it is strongly full), as can be seen from the above remarks. Also it follows from [2, Proposition 1.3] that $K$ has the Jordan-Hahn property with respect to $\mathbb{P}$.

We point out that the normal state space of a $W^{*}$-algebra, or a $J B W$-algebra, is always projective. The converse is false (see for example, [1, Proposition 6.11]). If $L=P(M)$, where $M$ is a $W^{*}$-algebra, or a $J B W$-algebra, without Type $I_{2}$ part then $K_{L}$ is projective, as can be seen by combining [4], [5] and [12] with [9]. We will be interested in converses of this proposition for more general lattices, $L$. To aid clarity and simplify the exposition we shall work with the whole of $K_{L}$, rather than convex subsets of it. More general conclusions can be drawn by making minor modifications to the arguments given.

In all that follows $L$ shall be a complete orthomodular lattice satisfying the conditions (1) and (2), $K_{L}$ shall be its ( $\sigma$-convex) set of completely additive probability measures and $V_{L}$ the associated base norm space. We shall identify $L$ with $\hat{L}$, its image in $V_{L}^{*}$ and, when $K_{L}$ is strongly full we shall also call $L$ strongly full. 


\section{Logics and States}

We observe that, given $\phi$ in $K_{L}$, condition (2) implies that the element $y=\wedge\{x ; \phi(x)=1\}$ is such that $\phi(y)=1$ (cf. [8, page 75]). We write $y=s_{L}(\phi)$, and call it the support of $\phi$ in $L$.

In essence, the following simple, but very useful, result is contained in [13], and its proof is similar to the usual one for $W^{*}$-algebras. However, as we were unable to find a reference for this result in the form which we need, we include a short argument.

Let us recall that an element $x$ of $L$ is said to satisfy the countable chain condition if every mutually disjoint family of elements in $L$, dominated by $x$, is countable. We also say that $L$ satisfies the countable chain condition if 1 satisfies the countable chain condition.

Lemma 1. Let $x$ be a non-zero element of L. Then $x$ satisfies the countable chain condition if and only if $x=s_{L}(\phi)$ for some $\phi$ in $K_{L}$.

Proof. Suppose that $x=s_{L}(\phi)$, for some $\phi$ in $K_{L}$, and let $\left(x_{\alpha}\right)_{\alpha \in I}$ be some mutually disjoint family of non-zero elements in $L$, dominated by $x$. The set $S_{n}=\{\alpha \in I$; $\left.\phi\left(x_{\alpha}\right) \geqq 1 / n\right\}$ contains at most $n$ elements. If $\phi\left(x_{\alpha}\right)=0$, then $x_{\alpha}=0$. Since this is impossible, $x_{\alpha} \in S_{n}$, for some $n$. So, $I$ is countable.

Conversely, suppose that $x$ satisfies the countable chain condition. There exists $\phi$ in $K_{L}$ with $\phi(x)=1$ (by condition (1)), so that $s_{L}(\phi) \leqq x$. Choose a maximal family $\left(\phi_{\lambda}\right)_{\lambda \in I}$, in $K_{L}$, for which the $s_{L}\left(\phi_{\lambda}\right)$ are mutually disjoint and dominated by $x$. By assumption, $I \subseteq N$. Accordingly, put $\rho=\Sigma \lambda_{n} \phi_{n}$, where $\lambda_{n} \geqq 0, \Sigma \lambda_{n}=1$. Then $\rho \in K_{L}$, and since maximality ensures that $x=\vee s_{L}\left(\phi_{n}\right)$, it follows that $x=s_{L}(\rho)$. This completes the proof.

We shall characterise projective $\sigma$-convex sets $K$ for which exposed points exist in the unit interval, $[0,1]$, of $A^{b}(K)$. As usual, $V$ represents the base norm space with base $K, V^{*}=A^{b}(K)$ and $\mathbb{P}$ is the set projective units of $A^{b}(K)$. We note that when $K$ is $\sigma$-convex the statement of Lemma 1 holds for $\mathbb{P}$ and $K$ (replacing $L$ and $K_{L}$ ). We are grateful to the referee for bringing our attention to the fact that Lemma 2 has occurred in a slightly different form in a preprint of Edwards and Rüttimann, [6]. Since our proof of Lemma 2 is both short and quite different from theirs, we give it here.

Lemma 2. Let $K$ be a projective, $\sigma$-convex set. Then the following are equivalent.

(i) $\exp [0,1]$ is non-empty,

(ii) $\exp [0,1]=\mathbb{P}$,

(iii) P satisfies the countable chain condition.

Proof. It will be convenient to write $s_{\mathbb{P}}(\phi)=s(\phi)$, for each $\phi$ in $K$. We recall that $a \in \exp [-1,1]$ if and only if there exists $\rho$ in $V,\|\rho\|=1$ which takes the value 1 on $[-1,1]$ only at the point $a$.

(i) $\Rightarrow$ (iii): Let $a \in \exp [0,1]$. Then $2 a-1 \in \exp [-1,1]$, and so there exists $\rho \in V$, of norm unity, such that $\rho(b)<\rho(2 a-1)=1$, for every $b$ in $[-1,1], b \neq a$. If $\rho \in K$, this implies that $a=1=s(\rho)$, and (iii) follows as in Lemma 1. Similarly if $\rho \in-K$. Suppose, then, that $\rho$ does not lie in $K$ or $-K$. 
It will follow, from [2, Proposition 1.3], that there exist $\sigma, \tau$ in $K, p$ in $\mathbb{P}$, and a real number $\lambda, 0 \leqq \lambda \leqq 1$, such that,

$$
\rho=\lambda \sigma-(1-\lambda) \tau, \quad \sigma(p)=\tau(1-p)=1 .
$$

Observe that,

$$
\sigma(s(\tau))=\tau(s(\sigma))=0, \quad \rho(s(\sigma)-s(\tau))=\rho(2 p-1)=1, \quad s(\sigma)-s(\tau)[-1,1] .
$$

Therefore, by the uniqueness property of $2 a-1, s(\sigma)-s(\tau)=2 p-1=2 a-1$. In particular, $p=a$. Moreover, since $s(\sigma) \leqq p, s(\tau) \leqq 1-p$, we see that,

$$
s(\sigma) \vee(1-p)=s(\sigma)+1-p=s(\tau)+p=s(\tau) \vee p,
$$

and that,

$$
s(\sigma)=p \wedge(s(\sigma) \vee(1-p))=p \wedge(s(\tau) \vee p)=p .
$$

It follows that, $s(\tau)=1-p$. This means that $\frac{1}{2}(\sigma+\tau)$ is faithful on $\mathbb{P}$. So, as in Lemma 1 , we see that $\mathbb{P}$ must satisfy the countable chain condition.

(iii) $\Rightarrow$ (ii): Suppose that $\mathbb{P}$ satisfies the countable chain condition, and let $p$ lie in $\mathbb{P}$, where $0 \neq p \neq 1$ (the lemma is obvious if $A^{b}(K)$ has no non-trivial projective units). Since $K$ is $\sigma$-convex, the argument used in Lemma 1 implies that there exist $\sigma, \tau$ in $K$ with $s(\sigma)=p$ and $s(\tau)=1-p$. Put $\rho=\frac{1}{2} \sigma-\frac{1}{2} \tau$. Then $1=\rho(2 p-1) \leqq$ $\|\rho\| \leqq 1$. So, $\|\rho\|=1$. We will show that $2 p-1$ is the only element in $[-1,1]$ which is sent to 1 by $\rho$, so that $2 p-1 \in \exp [-1,1]$, and consequently $p \in \exp [0,1]$.

Suppose that $a \in[-1,1]$ in $A^{b}(K)$ and that $\rho(a)=1$. Then, $\sigma(a)-\tau(a)=2$, implying that $\sigma(a)=1, \tau(a)=-1$. In other words, writing $b=\frac{1}{2}(a+1)$, we have $\sigma(1-b)=\tau(b)=0$. Hence $\sigma(r(1-b))=\tau(r(b))=0$. So, $p \leqq 1-r(1-b)$ and $1-p \leqq 1-r(b) .\left(^{*}\right)$ But, $1-r(1-b) \leqq b \leqq r(b)$. Therefore, $1-r(1-b)+1-r(b)=$ $(1-r(1-b)) \vee(1-r(b))=1$. Hence, $1-r(1-b)=b=r(b)$, and $\left(^{*}\right)$ gives $b=p$. Therefore, $a=2 p-1$ and (ii) follows. The trivial observation that (ii) implies (i) completes the proof of the lemma. We remark that $\sigma$-convexity was required only to show that (iii) implies (ii).

Since the normal state space, $K$, of a $W^{*}$-algebra, or a $J B W$-algebra, $M$, is projective and $\sigma$-convex and the projective units of $A^{b}(K)$ correspond exactly to the projections in $M$, [2, Proposition 3.1], we observe the following in passing (see also [7]).

Corollary 3. The following are equivalent for the $W^{*}$-algebra, or $J B W$-algebra, $M$.

(i) $\exp [0,1]$ is non-empty,

(ii) $\exp [0,1]=P(M)$ (the projections of $M)$

(iii) $M$ is $\sigma$-finite.

At this point it is convenient to recall that the elements $x, y$ of $L$ are said to be compatible if $x=(x \wedge y) \vee\left(x \wedge y^{\perp}\right)(=x \wedge y+x \wedge(1-y))$, and that $x$ is said to be central if it is compatible with all elements of $L$. The set of all central elements of $L$ is a complete Boolean sublattice, called the centre of $L$. See [8], for a discussion.

Given a projective convex set, $K$, the elements $p \in \mathbb{P}, a \in A^{b}(K)$ are said to be compatible in $A^{b}(K)$ if $P a+P^{\prime} a=a$, where $P$ is the (unique) $P$-projection of $A^{b}(K)$ 
determined by $P(1)=p$, and $P^{\prime}$ is its quasi-complement. Here, $p \in \mathbb{P}$ is said to be central in $A^{b}(K)$ if it is compatible with all elements of $A^{b}(K)$, and the set of all such elements is said to be the Boolean centre of $\mathbb{P}$.

As pointed out in [1, page 40], it follows from [1, Proposition 5.4], that elements of $\mathbb{P}$ are lattice-compatible if and only if they are compatible as elements of $A^{b}(K)$ and, consequently, the lattice centre of $\mathbb{P}$ contains the Boolean centre of $\mathbb{P}$. It is observed, [1, page 40], that the converse holds under certain additional "spectral" hypotheses. But these remarks were made in a slightly more general context that we have here. It turns out that the two notions of centre coincide without spectral conditions, when $K$ is projective. We have the following lemma.

Lemma 4. Let $K$ be a projective convex set. Then the lattice centre of $\mathbb{P}$ coincides with the Boolean centre of $\mathbb{P}$.

Proof. We have only to show that the lattice centre is contained in the Boolean centre. Let $p$ lie in the lattice centre of $\mathbb{P}$, and let $a$ be any element of $A^{b}(K)$. From [2, Proposition 1.7], it follows that the linear span of $\mathbb{P}$ is weak* dense in $A^{b}(K)$. Choose a net $\left(y_{\alpha}\right)$ in lin $(\mathbb{P})$ such that $y_{\alpha} \rightarrow a$ in the weak* topology. Consider a typical $y_{\alpha}=\Sigma \lambda_{i} q_{i}$, say, where the $q_{i} \in \mathbb{P}$. By remarks made in the preamble, $P q_{i}+P^{\prime} q_{i}=q_{i}$, for each $i$, and so $P y_{\alpha}+P^{\prime} y_{\alpha}=y_{\alpha}$, where $P$ is the $P$-projection corresponding to $p$. By definition, $P$ is weak* continuous, and therefore $P a+p^{\prime} a=a$. So, $p$ is compatible with every element of $A^{b}(K)$, and the proof is complete.

Henceforth we can, without ambiguity, refer to the centre of $\mathbb{P}$.

By placing geometric conditions on $K_{L}$, we can draw quite sharp conclusions.

Theorem 5. Let $K_{L}$ be a projective convex set. Then

(i) The centre of $L$ is contained in the centre of $\mathbb{P}$.

(ii) If $L$ is strongly full and satisfies the countable chain condition, then $L=\exp [0,1]=\mathbb{P}, L$ and $\mathbb{P}$ are isomorphic (as complete orthomodular lattices), and the centres of $L$ and $\mathbb{P}$ coincide (where $\mathbb{P}$ is the set of projective units of $A^{b}\left(K_{L}\right)$.)

Proof. (i) Let $x$ lie in the centre of $L$. Given $\phi \in K=K_{L}$, define a function $\phi_{x}: L \rightarrow R$, by $\phi_{x}(y)=\phi(x \wedge y)$, for every element $y$ in $L$.

Suppose that $\left(y_{\alpha}\right)$ is a mutually disjoint family of elements in $L$. Then $\left(x \wedge y_{\alpha}\right)$ is another mutually disjoint family and, by [8, Theorem 7], for example, $x \wedge\left(\vee y_{\alpha}\right)=\vee\left(x \wedge y_{\alpha}\right)$. Therefore,

$$
\phi_{x}\left(\vee y_{\alpha}\right)=\phi\left(x \wedge\left(\vee y_{\alpha}\right)\right)=\phi\left(\vee\left(x \wedge y_{\alpha}\right)\right)=\Sigma \phi\left(x \wedge y_{\alpha}\right)=\Sigma \phi_{x}\left(y_{\alpha}\right),
$$

so that $\phi_{x}$ is a completely additive positive measure on $L$.

Because $x$ is central, $y=x \wedge y+(1-x) \wedge y$, for each element $y$ in $L$, and we see that $\phi=\phi_{x}+\phi_{1-x}$. Note that $\phi=\phi_{x}$ if $\phi(x)=1$, and $\phi=\phi_{1-x}$ if $\phi(x)=0$. Suppose $0 \neq \phi(x) \neq 1$. Then,

$$
\phi=\phi(x) \phi_{1}+\phi(1-x) \phi_{2},
$$

where

$$
\phi_{1}=\phi(x)^{-1} \phi_{x}, \quad \phi_{2}=\phi(1-x)^{-1} \phi_{1-x}
$$


both of which lie in $K$.

Writing $F_{a}=\{\rho \in K ; \rho(a)=1\}$, for $a \in A^{b}(K)$, we see that we have shown that,

$$
K=c o\left(F_{x} \cup F_{1-x}\right)=c o\left(F_{1-r(1-x)} \cup F_{1-r(x)}\right) .
$$

Hence, if $\rho \in K$ annihilates the projective unit $1-r(1-x)+1-r(x)$, then $\rho=0$. It follows that $1-r(1-x)+1-r(x)=1$. Thus, since $1-r(1-x) \leqq x \leqq r(x)$, we conclude that $x=r(x) \in \mathbb{P}$. Further, it is easy to see that $K$ is a direct convex sum of $F_{x}$ and $F_{1-x}$, so that $F_{x}$ is a split face of $K$. This means that $x$ is a central projective unit of $\mathbb{P}$, by [1, Proposition 10.2] (see also [2, page 504]), so completing the proof of (i).

(ii) Observe that $r\left(\vee y_{\alpha}\right)=\vee_{p} r\left(y_{\alpha}\right)$, for any family of elements, $\left(y_{\alpha}\right)$, in $L$. Indeed, for all $\alpha, y_{\alpha} \leqq \vee y_{\alpha} \leqq r\left(\vee y_{\alpha}\right)$, so $r\left(y_{\alpha}\right) \leqq r\left(\vee y_{\alpha}\right)$, consequently, $\vee_{P} r\left(y_{\alpha}\right) \leqq r\left(\vee y_{\alpha}\right)$. Conversely, if $\rho \in K$ and $\rho\left(\vee_{P} r\left(y_{\alpha}\right)\right)=0$, then, $\rho\left(y_{\alpha}\right)=0$, hence $\rho\left(\vee y_{\alpha}\right)=0$ (using the remark made prior to Lemma 1$)$, and so $\rho\left(r\left(\vee y_{\alpha}\right)\right)=0$. Therefore, $r\left(\vee y_{\alpha}\right) \leqq$ $\vee_{P} r\left(y_{\alpha}\right)$. Thus, if $L$ and $\mathbb{P}$ are equal, they must be isomorphic as complete orthomodular lattices.

Suppose then that $L$ is strongly full and satisfies the countable chain condition. Then, by Lemma 1 , there is an element of $K$ which is faithful on $L$ which, by assumption, is also faithful on $A^{b}(K)^{+}$, and hence faithful on $\mathbb{P}$. Therefore, $\mathbb{P}$ has the countable chain condition and every element of $\mathbb{P}$ is of the form $s_{\mathbb{P}}(\phi)$, for some $\phi$ in $K$, as can be seen from the proof of Lemma 1 ( $K$ is $\sigma$-convex).

Thus, given any non-trivial projective unit, $p$, of $A^{b}(K)$, there exists $\phi$ in $K$ with support $1-p$. By assumption, since $\phi$ is not faithful on $A^{b}(K)$, there exists non-zero $x$ in $L$ with $\phi(x)=0$. But then, $\phi(1-r(x))=1$, and so, $1-p \leqq 1-$ $r(x) \leqq 1-x$. That is $0 \neq x \leqq p$. This means that if $z \in L$ and $r(z) \wedge_{P} r(1-z) \neq 0$, then there exists non-zero $y$ in $L$ such that $y \leqq r(z), y \leqq r(1-z)$. If $\psi \in K$ with $\psi(z)=0$, then $\psi(r(z))=0$, so $\psi(y)=0$, which implies $y \leqq z$, by condition (1). Similarly, $y \leqq 1-z$ and so $y=0$, a contradiction. Therefore, $r(z) \wedge{ }_{p} r(1-z)=0$ and, since $1-r(1-z) \leqq$ $r(z)$, the orthomodular identity on $\mathbb{P}$ implies that $r(z)=1-r(1-z)$, and hence that $z=r(z) \in \mathbb{P}$. In other words, $L \subseteq \mathbb{P}$ and moreover, the argument of the first paragraph implies that $L$ is a sublattice of $\mathbb{P}$. Now, using the fact that every non-zero element of $\mathbb{P}$ dominates some non-zero element of $L$, a simple Zorn's Lemma argument shows $L$ to be equal and isomorphic to $\mathbb{P}$, thereby completing the proof.

Even if we consider lattices, $L$, without Type $I_{2}$ part (cf. [4]), in this generality we have no reasonable hope of obtaining a generalisation of Gleason's Theorem with which to deduce a converse of Theorem 5(ii). However, we can re-cast Theorem 5(ii) into an "if and only if" form. For the sake of completeness we do so below.

By the projective units associated with a projective convex set $K$, we mean the lattice of projective units of $A^{b}(K)$.

Corollary 6. Let $S$ be a complete orthomodular lattice satisfying the countable chain condition. Then $S$ is isomorphic to the lattice of projective units associated with a projective, $\sigma$-convex set if and only if $K_{S}$ contains a projective, $\sigma$-convex and strongly full subset.

Proof. Suppose that $K$ is a strongly full and projective, $\sigma$-convex subset of $K_{S}$. Then, recalling the notation introduced in Sect. 1, we have $S \subseteq V(K)^{*} \simeq A^{b}(K)$, and 
$S$ is isomorphic (and equal) to the projective units of $A^{b}(K)$, by arguments given in the proof of Thoerem 5(ii).

The converse follows from the fact, observed in Sect. 1, that $K$ is a strongly full subset of $K_{\mathbb{P}}$, whenever $K$ is a projective convex set and $\mathbb{P}$ is the lattice of projective units of $A^{b}(K)$.

For the sake of completeness, at this point we ought to draw attention to the elementary fact that if $L$ is Boolean, then it can be identified with the lattice of all projections in a commutative von Neumann algebra. Indeed, suppose that $L$ is a complete Boolean algebra. Then $L$ can be realised as the characteristic functions of the clopen sets of some compact Stonean space, $S$ (the representation space of $L$ ). In addition, the conditions (1) and (2), imposed on $K_{L}$, ensure that $S$ has a separating family of normal measures, so that $S$ is hyperstonean. Therefore, $C[S]$ is the self-adjoint part of a commutative $W^{*}$-algebra and, by the above identification, its lattice of projections is $L$. We see that upon identifying (as we may) $K_{L}$ with the normal state space of $C[S]$, we may go on to identify $A^{b}\left(K_{L}\right)$ with $C[S]$ and conclude that $L=\mathbb{P}$, the lattice of $P$-projections of $A^{b}\left(K_{L}\right)$.

We mentioned previously that the Jordan-Hahn property is closely connected with strong fullness. As evidence for this we state:

Corollary 7. Let $K_{L}$ be projective. Then the following implications hold.

(a) If $K_{L}$ has the Jordan-Hahn property (with respect to $L$ ) then it is strongly full and ext $[0,1] \subseteq \bar{L}$ (the weak* closure of $L$ in $A^{b}\left(K_{L}\right)$ ).

(b) If L satisfies the countable chain condition, then the following are equivalent

(i) L is strongly full,

(ii) $L=\exp [0,1]$,

(iii) $K_{L}$ has the Jordan-Hahn property.

Proof. (a) Suppose that $K_{L}$ has the Jordan-Hahn property and that $\phi \in K_{L}=K$ is not faithful on $V_{L^{+}}^{*}=A^{b}(K)^{+}$. Then $\phi(a)=0$, for some non-zero $a \in V_{L_{+}}^{*}$, and so $\phi(r(a))=0$. Write $p=1-r(a)$, choose $\tau$ in $K$ with $\tau(1-p)=1$, and put $\rho=\frac{1}{2} \phi-\frac{1}{2} \tau$. Since $\quad 1=\rho(2 p-1) \leqq\|\rho\| \leqq 1, \quad\|\rho\|=\left\|\frac{1}{2} \phi\right\|+\left\|\frac{1}{2} \tau\right\|=1 . \quad$ Now, by the Jordan-Hahn property, there exist $\phi_{1}, \phi_{2}$ in $K$ and an $x$ in $L$ such that,

$$
\rho=\lambda \phi_{1}-\mu \phi_{2}, \quad \phi_{1}(x)=\phi_{2}(1-x)=1, \quad \text { where } \lambda, \mu \geqq 0 .
$$

But then, $\lambda+\mu \geqq\|\rho\| \geqq \rho(2 x-1)=\lambda+\mu$. Therefore, $\lambda+\mu=1$ and, by the uniqueness property of the decomposition of $\rho$ (see [2 Proposition 1.3]), it follows that $\phi=\phi_{1}$. Therefore since $x \neq 1$ and $\phi_{1}(x)=1, \phi$ is not faithful on $L$. This proves that $L$ is strongly full. The last statement in (a) follows from Milman's Theorem, since for each $\rho$ in $V_{L},\|\rho\|=1$, there exists $x$ in $L$ for which $\rho(2 x-1)=1$.

(b) The implications (iii) $\Rightarrow$ (i) $\Rightarrow$ (ii) follow from part (a) and Theorem 5(ii), respectively. As for the implication (ii) $\Rightarrow$ (iii): if $L=\exp [0,1]$, then $L$ equal the projective units of $A^{b}\left(K_{L}\right)$, by Lemma 2 , and (iii) is now an immediate consequence of $[2$, Corollary 1.3].

As our penultimate observation, we will show how to extend to a lattice $L$ without the countable chain condition, the second part of Theorem 5 . 
Consider a lattice, $L$, (as always, satisfying the standing assumptions) with $K_{L}=K$ projective, and let $S$ be a subset of $V_{L}^{*}\left(=A^{b}(K)\right)$. Denote by $F(S)$ the semi-exposed face of $V_{L}^{*}$ generated by $S$. Of course, $V_{L}^{*}=F(L)$. We write $L[0, x]$ for the complete orthomodular lattice $\{y \in L: y \leqq x\}$.

We shall call $L$ locally full when the following condition is satisfied: If $x \in L$ and $\phi \in K$ is faithful on $L[0, x]$, then $\phi$ is faithful on $F(L[0, x])$.

Let us observe that local fullness is equivalent to strong fullness if $L$ satisfies the countable chain condition and $K_{L}$ is projective. On the one hand, local fullness clearly implies that $K_{L}$ is strongly full. Conversely, if $K_{L}$ is strongly full and $L$ satisfies the countable chain condition then $L=\mathbb{P}$, by Theorem 5(ii), and we see that $L$ is locally full because every semi-exposed face of $A^{b}\left(K_{L}\right)$ is of the form $\mathrm{Im}^{+} P$, for some $P$-projection, $P$, of $A^{b}\left(K_{L}\right)$ (see the proof of [2, Proposition 1.1]).

Theorem 8. Let $K_{L}=K$ be projective, and let $L$ be locally full. Then $L$ may be identified with the lattice of projective units, $\mathbb{P}$, of $A^{b}(K)$.

Proof. Let us first make a general observation: given a non-zero element $x$ in $L$, there exists $\phi$ in $K$ for which $\phi(x)=1$. Thus, $s_{L}(\phi) \leqq x$, and we see from Lemma 1 that $x$ dominates a nontrivial element, of $L$, and satisfies the countable chain condition. So, letting $\left(x_{\alpha}\right)$ be a maximal disjoint family of elements in $L$, such that $x_{\alpha} \leqq x$ and $x_{\alpha}$ satisfies the countable chain condition, for all $\alpha$, we see that $x=\vee x_{\alpha}$.

Now suppose that $L$ is locally full, and let $x$ be a non-zero element, in $L$, satisfying the countable chain condition. By Lemma $1, x=s_{L}(\phi)$, for some $\phi$ in $K$. Then $\phi$, being faithful on $L[0, x]$, is faithful on $F(L[0, x])$, by assumption. Note that $r(x) \in F(L[0, x])$ : in fact, by the proof of [2, Proposition 1.1], $F(L[0, x])=$ $\operatorname{Im}^{+} P$, where $P$ is the $P$-projection on $A^{b}(K)$ for which $P(1)=r(x)$. But then $r(x)-(1-r(1-x)) \in F(L[0, x])$ and, since $\phi(x)=1$, we have that $\phi(r(x)-$ $(1-r(1-x)))=0$. Therefore, $r(x)=1-r(1-x)$, so $x=r(x) \in \mathbb{P}$. Combining this with the first paragraph, above, and the argument in the first paragraph of the proof of Theorem 5(ii), we see that $L$ is contained in $\mathbb{P}$ and is a sublattice of $\mathbb{P}$.

Finally, let $\phi \in K$ and observe that $s_{P}(\phi) \leqq 1-r\left(1-s_{L}(\phi)\right) \leqq s_{L}(\phi) \in \mathbb{P}$. Using Lemma 1, and repeating the above argument, we see that $\phi$ is faithful on the interval $\mathbb{P}\left[0, s_{L}(\phi)\right]$ of $\mathbb{P}$. Consequently, $s_{P}(\phi)=s_{L}(\phi) \in L$. It now follows that $L$ is equal and isomorphic to $\mathbb{P}$, by applying to $\mathbb{P}$, the argument of the first paragraph, so completing the proof.

Theorem 8 can be re-cast as an "if and only if" just as in Corollary 6. In a similar way, replacing $K_{L}$ in each of the statements with an appropriate convex subset having appropriate geometric properties, most of these results admit generalisations. As these will be apparent to the reader, we omit the details.

We would like to record one final result, however. It follows from Proposition 8, Gleason's Theorem for $W^{*}$-algebras [5], [12], and that for $J B W^{*}$-algebras, [3], together with $[9$, Theorems $1.5,2.10]$. The meaning of a lattice without Type $I_{2}$ part was described in [4].

Corollary 9. Suppose that $L$ has no Type $I_{2}$ part. Then $L$ is isomorphic to

(a) The lattice of all projections in a JBW-algebra if and only if $K_{L}$ is spectral, elliptic and $L$ is locally full. 
(b) The lattice of all projections in a $W^{*}$-algebra if and only if $K_{L}$ is spectral, elliptic, has the global 3-ball property, and L is locally full.

Acknowledgement. It is a pleasure to thank Dr. Pavel Pták for sharing with us his extensive knowledge of quantum logics, and the Royal Society who paid for his stay in Reading under their Guest Fellowship Scheme. We gratefully acknowledge financial support from the S.E.R.C. under contract GR/C/64035.

\section{References}

1. Alfsen, E. M., Shultz, F. W.: Non-commutative spectral theory for affine function spaces on convex sets. Mem. Am. Math. Soc. 1976

2. Alfsen, E. M., Shultz, F. W.: On non-commutative spectral theory and Jordan algebras. Proc. London Math. Soc. (3), 38, 497- 516 (1979)

3. Bunce, L. J., Wright, J. D. M.: Quantum measures and states on Jordan algebras. Commun. Math. Phys. 98, 187-202 (1985)

4. Bunce, L. J., Wright, J. D. M.: Quantum logic, state space geometry and operator algebras. Commun. Math. Phys. 96, 345- 348 (1984)

5. Christensen, E.: Measures on projections and physical states. Commun. Math. Phys. 86, 529- 538 (1982)

6. Edwards, C. M., Rüttiman, G. T.: On the facial structure of the unit balls in a GL-space and its dual. Preprint

7. Edwards, C. M., Rüttiman, G. T.: On the unit balls in a $J B W$-algebra and its predual. Preprint

8. Holland, S. S., Jr.: A Radon-Nykodim theorem for dimension lattices. Trans. Am. Math. Soc. 108, 67-87 (1963).

9. Iochum, B., Shultz, F. W.: Normal state spaces of Jordan and von Neumann algebras. J. Funct. Anal. 50, 317-328 (1983)

10. Rüttiman, G. T.: Jordan-Hahn decomposition of signed weights on finite orthogonality spaces. Commun. Math. Helvetici 52, 129-144 (1977)

11. Wilbur, W. J.: On characterising the standard quantum logics. Trans. Am. Math. Soc. 233, 265-282 (1977)

12. Yeadon, F. J.: Measures on projections in $W^{*}$-algebras of Type II. Bull. London Math. Soc. 15, 139$145(1983)$

13. Zierler, N.: Axioms for non-relativistic quantum mechanics. Pac. J. Math. 11, 1151-1169 (1961)

Communicated by H. Araki

Received August 10, 1984; in revised form January 7, 1985 